\title{
BMJ Open Study protocol for a randomised controlled trial investigating two different refeeding formulations to improve safety and efficacy of hospital management of adolescent and young adults admitted with anorexia nervosa
}

\author{
Elizabeth Parker (D) , ${ }^{1,2}$ Victoria Flood (D) , 2,3 Mark Halaki, ${ }^{2}$ Christine Wearne, ${ }^{4}$ \\ Gail Anderson, ${ }^{5}$ Linette Gomes, ${ }^{5}$ Simon Clarke, ${ }^{5,6,7}$ Frances Wilson, ${ }^{8}$ \\ Janice Russell, ${ }^{7,9}$ Elizabeth Frig, ${ }^{10}$ Michael Kohn ${ }^{5,6,7}$
}

To cite: Parker E, Flood V, Halaki M, et al. Study protocol for a randomised controlled trial investigating two different refeeding formulations to improve safety and efficacy of hospital management of adolescent and young adults admitted with anorexia nervosa. BMJ Open 2020;10:e038242. doi:10.1136/ bmjopen-2020-038242

- Prepublication history and additional material for this paper are available online. To view these files, please visit the journal online (http://dx.doi. org/10.1136/bmjopen-2017018426).

Received 04 March 2020 Revised 17 August 2020 Accepted 02 September 2020

D) Check for updates

(c) Author(s) (or their employer(s)) 2020. Re-use permitted under CC BY-NC. No commercial re-use. See rights and permissions. Published by BMJ.

For numbered affiliations see end of article.

Correspondence to Elizabeth Parker; elizabeth.parker@health.nsw. gov.au

\begin{abstract}
Introduction Providing effective nutritional rehabilitation to patients hospitalised with anorexia nervosa (AN) is challenging, partly due to conservative recommendations that advocate feeding patients at low energy intakes. An 'underfeeding syndrome' can develop when patients are not provided with adequate nutrition during treatment, whereby malnourished patients fail to restore weight in a timely matter, and even lose weight. Of particular concern, the reintroduction of carbohydrate in a starved patient can increase the risk of developing electrolyte, metabolic and organ dysfunction. The proposed trial assesses the efficacy and safety of a lower carbohydrate enteral formula ( $28 \%$ carbohydrate) against a standard enteral formula (54\% carbohydrate), in adolescent and young adult patients (aged 15-25 years), hospitalised with AN.
\end{abstract}

Methods and analysis The study employs a doubleblind randomised controlled trial design. At admission to hospital, malnourished adolescent and young adults with AN will be randomly allocated to commence feeding on a standard enteral feeding formula (1.5 $\mathrm{kcal} / \mathrm{mL}, 54 \%$ carbohydrate) or a lower carbohydrate isocaloric enteral feeding formula $(1.5 \mathrm{kcal} / \mathrm{mL}, 28 \%$ carbohydrate). Assessments of nutritional intake, weight and biochemistry (phosphate, magnesium, potassium) will be conducted at baseline and during the first 3 weeks of hospital admission. The primary outcome measure will be incidence of hypophosphatemia. Secondary outcomes include weight gain, oedema, other electrolyte distortion, length of hospital admission, admission to the Intensive Care Unit (ICU) and number of days to reach medical stability, using defined parameters.

Ethics and dissemination The protocol was approved by the Western Sydney Local Health District Human Research Ethics Committee and institutional research governance approvals were granted. Written informed consent will be sought prior to study enrolment. Study findings will be widely disseminated through peer-reviewed publications and conference presentations.
Strengths and limitations of this study

- In terms of comparing the two feeding formulas, use of a double-blinded randomised controlled trial design will control for bias, thereby enhancing the reliability of the study findings.

- By employing the participant group hospitalised young people with anorexia nervosa (AN), the study addresses the urgent need to identify effective treatments to reduce length of hospital stay and in the longer term prevent chronicity.

- To the authors knowledge, this is the first study comparing a standard enteral feeding formula against a lower carbohydrate formula in patients with AN.

Trial registration number Australian New Zealand Clinical Trials Registry (ACTRN12617000342314); Preresults.

\section{INTRODUCTION}

Patients hospitalised with anorexia nervosa (AN) require (1) acute management, (2) restoration of positive caloric balance and (3) nutritional rehabilitation to reverse the medical complications of malnutrition, such as dehydration, bradycardia, hypotension, hypothermia, neutropenia, hypertransaminaemia, amenorrhoea and impairments in growth and bone heath. ${ }^{1}$

Malnourished patients, such as those with $\mathrm{AN}$, are considered at high risk for the development of refeeding syndrome during nutritional rehabilitation. Refeeding syndrome lacks a consistent definition in the literature; however, it is generally described as severe electrolyte disturbances (in particular phosphate) leading to clinical deterioration and 
possible sudden cardiac death, and can occur when a starved person undergoes nutrition repletion. ${ }^{2}$

During starvation, the body is in a catabolic state and shifts from carbohydrate to fat and protein utilisation. This results in an altered insulin response and increased glucagon secretion. ${ }^{3}$ When the body undergoes nutrition repletion, the change from catabolic to anabolic metabolism results in switching back from fat utilisation to carbohydrate utilisation. This process results in lower serum levels of phosphate. Changes with refeeding can progress to clinical symptoms, including acute circulatory fluid overload, respiratory failure and cardiac failure, and result in death. Hypophosphatemia, in particular, is seen as the precursor to the development of refeeding syndrome. ${ }^{3}$

Consensus-based guidelines for providing nutritional rehabilitation to patients with AN vary; however, most guidelines advocate feeding patients slowly to prevent refeeding complications, such as refeeding syndrome. For example, the UK-based guidelines recommend initial feeding rates as low as $10 \mathrm{kcal} / \mathrm{kg} /$ day for at risk patients and $5 \mathrm{kcal} / \mathrm{kg} /$ day for extreme cases (body mass index $\left.(\mathrm{BMI})<14 \mathrm{~kg} / \mathrm{m}^{2}\right),{ }^{4}$ the American-based guidelines recommend feeding at a slightly higher rate of 30-40 $\mathrm{kcal} / \mathrm{kg} /$ day $(1000-1600 \mathrm{kcal} / \text { day })^{5}$ and Australian-based guidelines advocate a starting rate of $6000 \mathrm{~kJ} /$ day $(1435$ $\mathrm{kcal} /$ day $){ }^{6}$

Irrespective of which guidelines are employed, slow feeding results in poor weight gain, and typically initial weight loss in an already malnourished patient group. ${ }^{7}$ Under current guidelines, the rate of feed is not increased if a patient displays electrolyte derangement. ${ }^{4}$ This often results in 'underfeeding syndrome', whereby patients do not reach adequate energy intakes to restore weight in a timely manner, which in turn potentially delays correction of medical instability, prolongs hospital stay and increases the associated costs of hospital treatment. ${ }^{8}$ Of concern, strict adherence to guidelines has not prevented the occurrence of refeeding syndrome in a variety of clinical areas, including AN. This calls into question the validity of these conservative guidelines, ${ }^{9-11}$ and points to a need for them to be re-evaluated and potentially revised.

A growing body of evidence is now challenging the 'start low, go slow' approach advocated in conservative guidelines. A systematic review by Garber et al included 27 studies investigating approaches to refeeding patients with AN. ${ }^{12}$ The authors concluded that higher caloric refeeding had not been associated with increased risk for the development of refeeding syndrome under close medical monitoring and electrolytes correction. ${ }^{12}$ There was insufficient evidence to change the current standard of care for refeeding severely malnourished inpatients, defined as BMI $<15 \mathrm{~kg} / \mathrm{m}^{2}$ in adults and $<70 \%$ of median BMI in adolescents. ${ }^{12-14}$ However, at present, consensus is lacking around what is considered the current standard of care.

The UK-based National Institute for Clinical Excellence guidelines recommending starting 'at risk' patients on $5-10 \mathrm{kcal} / \mathrm{kg} /$ day were originally published more than a decade ago, and the updated version in 2017 has continued to recommend the same approach of slowly introducing nutrition. ${ }^{4}$ The Management of Really Sick Patients with Anorexia Nervosa report for refeeding has recommended more frequent assessment (at least 12 hourly) so that energy provision can be increased in the absence of refeeding syndrome, in order to minimise the risk of 'underfeeding syndrome'. ${ }^{13}$

While new evidence supports more aggressive feeding in adolescent patients with $\mathrm{AN}$, less robust evidence exists for adult patients with AN. ${ }^{12}$ More recent recommendations have been published stating inpatient refeeding protocols for adolescent patients can be 'more aggressive than previously recommended', however these lack specific recommendations for the initial caloric prescription. ${ }^{14}$ Some authors have recommended starting caloric intakes as high as $35-45 \mathrm{kcal} / \mathrm{kg} /$ day (Cape Town Metropole Paediatric Interest Group ${ }^{15}$ ) to $60 \mathrm{kcal} / \mathrm{kg} /$ day (Afzal $e t a l^{16}$ ) in adolescents with AN, $1600 \mathrm{kcal} /$ day in adults with AN. ${ }^{17}$ Adult patients with AN have been safely started on caloric intakes of 1200-1500 kcal/day, without the occurrence of refeeding syndrome, even in populations with a $\mathrm{BMI}<12 \mathrm{~kg} / \mathrm{m}^{2} .{ }^{1819}$

Of particular concern is the reintroduction of carbohydrate in a starved patient, which is postulated to lead to a surge in insulin and result in electrolyte derangement and increase the risk of developing refeeding complications. ${ }^{20}$ While the literature suggests strategies to avoid refeeding complications such as continuous delivery of nutrients (eg, nasogastric feeding) with less than $40 \%$ of energy from carbohydrate, ${ }^{3}$ this has not been formally tested. Furthermore, standard enteral formula usually contain $>50 \%$ energy from carbohydrate. ${ }^{21}$ Not all studies demonstrating the safety of higher energy intakes (initiating $\geq 1200 \mathrm{kcal} /$ day) have specified the macronutrient composition provided to patients during treatment. ${ }^{18} 1922-24$ Studies that have specified the macronutrient content have ranged from 1200 to $1764 \mathrm{kcal} /$ day and $40 \%-60 \%$ carbohydrate in oral mealbased programmes ${ }^{825-28}$ and $1617-2400 \mathrm{kcal} /$ day and $44 \%-50 \%$ carbohydrate in programmes utilising nasogastric feeding. ${ }^{29} 30$

The current trial aims to test the efficacy and safety of a lower carbohydrate enteral formula (less than $40 \%$ carbohydrate) against a standard enteral formula (54\% carbohydrate), in adolescent and young adult patients (aged 15-25 years), hospitalised with AN.

Our working hypothesis is that carbohydrate ingested during a starving state increases the risk of hypophosphatemia, via a process whereby a surge of insulin following carbohydrate ingestion causes an intracellular shift of glucose, fluid and electrolytes. Hence, it is predicted that malnourished patients with AN who are provided with a low carbohydrate enteral formula will have lower rates of hypophosphatemia compared with malnourished patients with AN provided with a standard higher carbohydrate enteral formula. 
Secondary predictions are that, relative to patients on a standard enteral formula, patients provided with a lower carbohydrate formula may reach feeding target rates sooner due to less electrolyte derangement, which can delay the increase in the rate of feeds. This will affect total energy intake, rate of weight gain, number of days to reach medical stability and length of hospital stay (LOS).

\section{METHODS AND ANALYSIS \\ Study design}

This study will use a double-blind randomised controlled design. Adolescent and young adult participants will be randomised into two groups (Standard Feed vs Lower Carbohydrate Feed), using a computer-generated sequence placed into opaque envelopes, stratified by gender. This trial protocol was prepared based on the SPIRIT 2013 statement (online supplemental file 1). ${ }^{31}$

\section{Recruitment}

Participants will be recruited at two public hospitals in NSW with specialised inpatient eating disorder treatment services. Participants will be invited to participate at the commencement of their inpatient treatment, and will be approached once the treating team has decided the patient requires nasogastric feeding. The assessment for the requirement of nasogastric feeding will be made by the treating medical officer based on clinical need and will be made independent to the study. Written informed consent (online supplemental file 2) will be obtained from all participants and will be obtained from guardians if under 18 years of age.

\section{Participants}

This study will recruit patients (aged 15-25 years) hospitalised with AN (assessed by the supervising medical officer using DSM-5 criteria), ${ }^{32}$ and requiring nasogastric feeding for nutritional rehabilitation.

Exclusion criteria will include patients that have transferred from another facility, where they have already received nasogastric feeding and/or prophylactic phosphate replacement.

\section{Enteral feed content}

The standard and trial enteral feed were products of Abbott Nutrition, and the nutrient breakdown is described in table 1.

\section{Treatment}

The flowsheet of the treatment protocol is described in figure 1 .

\section{Supplementation}

Participants will receive a daily multivitamin. Participants will not receive prophylactic phosphate supplementation if serum phosphate levels are $>1.0 \mathrm{mmol} / \mathrm{L}$. Participants will receive $1 \mathrm{~g}$ phosphate supplementation prior to commencing nasogastric feeds if baseline serum phosphate level is $\leq 1.0 \mathrm{mmol} / \mathrm{L}$, and will be provided

\begin{tabular}{|c|c|c|}
\hline Feed & $\begin{array}{l}\text { Standard } \\
1.5 \mathrm{kcal} / \mathrm{mL} \\
\text { formula }\end{array}$ & $\begin{array}{l}\text { Lower } \\
\text { carbohydrate } 1.5 \\
\text { kcal/mL formula }\end{array}$ \\
\hline Energy (kcal) & 1500 & 1500 \\
\hline Carbohydrate (g) & 204 (54\% energy) & 106 (28\% energy) \\
\hline Fat (g) & 49 (29\% energy) & 93 (56\% energy) \\
\hline Protein (g) & 63 (17\% energy) & 63 (17\% energy) \\
\hline Fibre (g) & 0 & 0 \\
\hline $\mathrm{Na}(\mathrm{mg})$ & 1400 & 1310 \\
\hline Potassium (mg) & 1650 & 1960 \\
\hline Calcium (mg) & 1000 & 1050 \\
\hline Phosphorus (mg) & 1000 & 1050 \\
\hline Magnesium (mg) & 310 & 422 \\
\hline Iron (mg) & 22 & 19 \\
\hline Zinc (mg) & 19 & 24 \\
\hline Manganese (mg) & 5.0 & 5.5 \\
\hline Folic acid $(\mu \mathrm{g})$ & 400 & 840 \\
\hline Vitamin $B_{12}(\mu \mathrm{g})$ & 4 & 13 \\
\hline Thiamine (mg) & 2.6 & 3.2 \\
\hline $\begin{array}{l}\text { Osmolality (mOsm/ } \\
\mathrm{kg} \mathrm{H}_{2} \mathrm{O} \text { ) }\end{array}$ & 510 & 475 \\
\hline
\end{tabular}

with phosphate supplementation if serum phosphate levels are $\leq 1.0 \mathrm{mmol} / \mathrm{L}$ during nutritional rehabilitation. Participants will not receive prophylactic magnesium or potassium supplementation; however, electrolytes will be monitored closely and supplementation will be provided if clinically indicated.

\section{Assessments}

Assessments of primary and secondary outcomes will be conducted during the first 3 weeks of hospital admission. Participation will cease on discharge.

\section{Diagnosis of AN}

Diagnosis based on clinical assessment by the supervising medical officer, using DSM-5 criteria. $^{32}$

\section{Nutrition assessment}

An accredited, practising dietitian will complete a nutrition assessment using the validated nutrition assessment tool, the Subjective Global Assessment (SGA). ${ }^{33}$ The assessment examines known prognostic indicators of nutritional status, such as change in weight, change in oral intake, gastrointestinal symptoms, change in functional capacity and the presence of muscle wasting and loss of subcutaneous fat stores. The nutritional status of participants will be scored as an SGA rating A (well nourished), B (mildly/moderately malnourished) or C (severely malnourished).$^{33}$ In addition, nutritional status will be assessed in patients using percentage median BMI (\% $\mathrm{mBMI}$ ), defined as $80 \%-90 \%$ mBMI (mild 


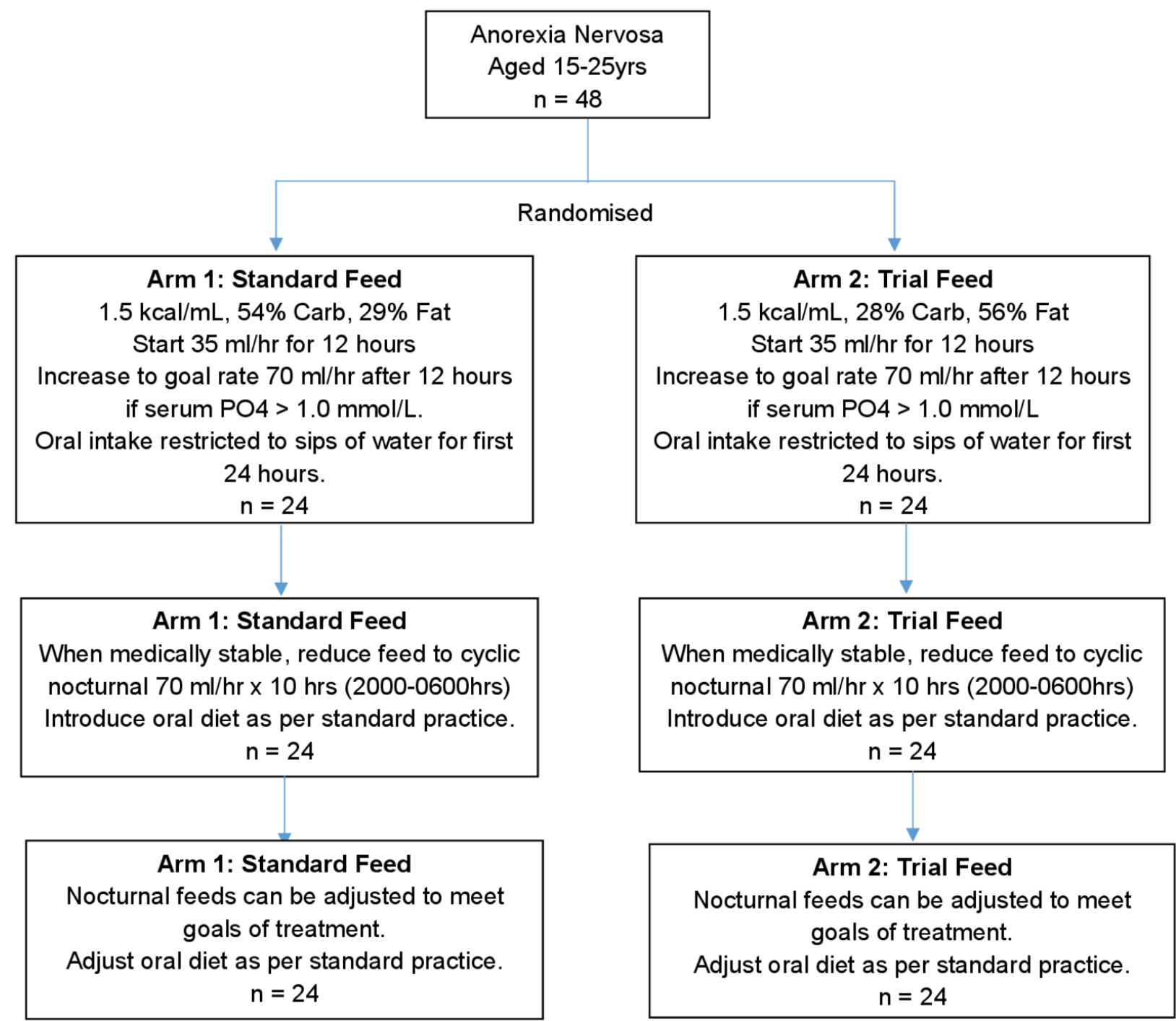

Figure 1 Flowchart of treatment protocol.

malnutrition), $70 \%-79 \%$ mBMI (moderate malnutrition) or $<70 \% \mathrm{mBMI}$ (severe malnutrition). ${ }^{14}$

Anthropometric measures will be taken at baseline by a trained accredited practicing dietitian and will include: height using a wall mounted stadiometer, skinfold measurement using callipers (Holtain, Crymych, UK) and nondominant hand-grip strength using a dynamometer (Jamar, Sammons Preston Roylan, Bolingbrook, Illinois, USA), with technique recommended in practice. Standard percentile ranges for participant's sex and age will be used for comparison of skinfold measurement. ${ }^{34}{ }^{35}$ Percentage of normal grip strength will be compared with normative data using z scores. ${ }^{36}$ Body mass will be recorded from routine weight checks, minimum 3 days/week, using the ward scales recorded to the nearest $0.1 \mathrm{~kg}$, and BMI will be calculated using the initial measured height.

\section{Blood tests}

Electrolytes (sodium, phosphate, magnesium, potassium) will be measured at baseline, 6-8 hours after starting feeds, then daily for the first week and at least weekly in week 2 and week 3 . Thiamine and liver function tests will be monitored at baseline and weekly during the first 3 weeks of admission. Blood glucose levels will be measured at baseline and at least two times per day during the first week and at least weekly in week 2 and week 3 .

\section{Dietary intake}

The nutritional intake of participants will be assessed by an accredited practising dietitian, using recorded food charts, standardised meal plans and recorded administered enteral feed volumes. Participants on oral intake will have three main meals and three mid meals supervised by nursing staff as per routine care. The macronutrient content of oral diet will also be monitored and reported. Patients on nocturnal nasogastric feeds will receive feeds over 10 hours (20:00-06:00).

\section{Medical record review}

Participant medical records will be reviewed to generate the following information: standard demographics, LOS, outcomes from daily medical monitoring, presence of peripheral oedema, electrolyte replacement, admission to ICU, days required to reach medical stability, defined 
as heart rate $>50$ beats per minute, temperature $>35.5^{\circ} \mathrm{C}$, blood pressure $>80 / 50 \mathrm{~mm} \mathrm{Hg}$ and postural hypotension $<20 \mathrm{~mm}$. Routine nursing monitoring will include heart rate, blood pressure, respiratory rate and blood glucose levels.

\section{Sample size}

The target sample size for this trial will be 48 . This power calculation was calculated using a dichotomous endpoint two independent study sample, incidence of $45 \%$ hypophosphatemia in the standard feed $\mathrm{arm}^{27}$ and $10 \%$ hypophosphatemia in the trial feed arm, 48 patients (24 in each arm) would have $80 \%$ power, alpha 0.05 . The power calculation was based on reported findings from a meal-based programme ${ }^{27}$ providing an initial energy intake and macronutrient composition comparable with the standard feed arm of this trial. Studies utilising nasogastric feeding could not be used in the power analysis due to the lack of reported macronutrient composition ${ }^{19} 23$ or the provision of prophylactic phosphate supplementation from commencement of treatment. ${ }^{29} 30$

\section{Outcome measures}

The primary outcome measure will be the incidence of hypophosphatemia.

Secondary outcome measures will be the change in weight, LOS, hypoglycaemia, development of peripheral oedema, serum thiamine (vitamin $\mathrm{B}_{1}$ ), prescribed electrolyte replacement, admission to ICU, days required to reach medical stability, defined as heart rate $>50$ beats per minute, temperature $>35.5^{\circ} \mathrm{C}$, blood pressure $>80 / 50$ $\mathrm{mm} \mathrm{Hg}$ and postural hypotension $<20 \mathrm{~mm}$.

\section{Statistical analysis}

Continuous outcomes will be assessed for normality and if normally distributed, the two treatment groups will be compared using repeated measures analysis of variance for variables with multiple time point measures (eg, change in weight over time) or independent t-tests for single measures (eg, LOS). Binary outcomes will be compared using a $\chi^{2}$ test. An interim analysis will be completed at midpoint.

\section{Auditing}

A coordinating study investigator will conduct regular site visits during recruitment to ensure the protocol is being followed. As per conditions of ethics approval, the human research ethics committee (HREC) is able to conduct independent audits as required.

\section{Reporting of adverse events}

Adverse events will be reported to the Australia New Zealand Clinical Trials Registry and the Western Sydney Local Health District HREC. Unblinding will occur at request of the treating medical team in the event of an adverse reaction (eg, allergic reaction).

\section{Patient and public involvement}

Patients or the public were not involved in the design or conduct plans of our research. Once the trial has been published, we intend to disseminate the main results to trial participants and will seek patient and public involvement in the development of an appropriate method of dissemination.

\section{DISCUSSION}

This study addresses the challenges of providing effective nutritional rehabilitation to adolescent and young adult patients hospitalised with AN, and will provide key evidence-based outcomes to inform changes to current consensus-based guidelines.

It is anticipated that the results of this trial will: (1) be translatable widely into clinical settings, (2) make a significant contribution to dietetic clinical practice, (3) inform the medical management of adolescent and young adult patients with AN, (4) inform the clinical practice of dietitians working with eating disordered patients, (5) improve the nutritional care and health of patients hospitalised with AN and (6) reduce LOS and associated costs.

\section{Ethics and dissemination}

This study protocol has received ethical approval from the Western Sydney Local Health District HREC. Approval has also been received from the Western Sydney Local Health District Research Governance and Sydney Local Health District Research Governance.

Participants will receive nutritional rehabilitation similar to what they would receive as part of standard care, with exception to the possibility of being randomised to receive an isocaloric enteral formula with a different macronutrient composition. As part of routine care, participants will receive close monitoring of electrolytes, and replacement if required, as well as adjustments to caloric intake required to promote adequate weight gain.

Study findings will be widely disseminated through peer-reviewed publications and conference presentation.

\section{Author affiliations}

${ }^{1}$ Department of Dietetics \& Nutrition, Westmead Hospital, Westmead, New South Wales, Australia

${ }^{2}$ Sydney School of Health Sciences, Faculty of Medicine and Health, The University of Sydney, Sydney, New South Wales, Australia

${ }^{3}$ Allied Health Research Unit, Western Sydney Local Health District, Westmead Hospital, New South Wales, Australia

${ }^{4}$ Department of Medical Psychology, Westmead Hospital, Westmead, New South Wales, Australia

${ }^{5}$ Department of Adolescent \& Young Adult Medicine, Westmead Hospital, Westmead, New South Wales, Australia

${ }^{6}$ Centre for Research into Adolescents' Health (CRASH); Department of Adolescent \& Young Adult Medicine, Westmead Hospital, Westmead, New South Wales, Australia

${ }^{7}$ Sydney School of Medicine, Faculty of Medicine and Health, The University of Sydney, Sydney, New South Wales, Australia

${ }^{8}$ Department of Psychiatry, Westmead Hospital, Westmead, New South Wales, Australia

${ }^{9}$ NSW Statewide Eating Disorder Service, Peter Beumont Unit, Professor Marie Bashir Centre, Royal Prince Alfred Hospital, Camperdown, New South Wales, Australia 
${ }^{10}$ Department of Nutrition and Dietetics, Royal Prince Alfred Hospital, Camperdown, New South Wales, Australia

\section{Twitter Victoria Flood @FloodVicki}

Contributors Development of the manuscript was led by EP. Funding was secured by EP, VF, MK, FW, SC and EF. The study design was developed by EP, VF, MK and FW and further refined by LG, GA, SC, MH, CW, EF and JR. Sample size calculation was determined by VF. Ethics approval and amendments were obtained by EP, VF and MK, with assistance from MH and CW. The trial will be coordinated by EP. All authors approved the final version of the manuscript.

Funding This work was supported by NSW Ministry of Health Translational Research Grants Scheme grant number 038.

Competing interests None declared.

Patient and public involvement Patients or the public were not involved in the design or conduct plans of our research. Once the trial has been published, we intend to disseminate the main results to trial participants and will seek patient and public involvement in the development of an appropriate method of dissemination.

Patient consent for publication Not required.

Provenance and peer review Not commissioned; externally peer reviewed.

Open access This is an open access article distributed in accordance with the Creative Commons Attribution Non Commercial (CC BY-NC 4.0) license, which permits others to distribute, remix, adapt, build upon this work non-commercially, and license their derivative works on different terms, provided the original work is properly cited, appropriate credit is given, any changes made indicated, and the use is non-commercial. See: http://creativecommons.org/licenses/by-nc/4.0/.

\section{ORCID iDs}

Elizabeth Parker http://orcid.org/0000-0002-4299-6091

Victoria Flood http://orcid.org/0000-0001-5310-7221

\section{REFERENCES}

1 Brown C, Mehler PS. Medical complications of anorexia nervosa and their treatments: an update on some critical aspects. Eat Weight Disord 2015;20:419-25.

2 Friedli N, Stanga Z, Sobotka L, et al. Revisiting the refeeding syndrome: results of a systematic review. Nutrition 2017;35:151-60.

3 Kohn MR, Madden S, Clarke SD. Refeeding in anorexia nervosa: increased safety and efficiency through understanding the pathophysiology of protein calorie malnutrition. Curr Opin Pediatr 2011;23:390-4.

4 National Institute for Clinical Excellence (NICE). Nutrition support in adults: oral nutrition support, enteral tube feeding and parenteral nutrition, NICE clinical guideline 32, National Institute for health and clinical excellence. London, UK, 2006.

5 American Psychiatric Association. Practice guideline for the treatment of patients with eating disorders. 3rd edn. American Psychiatric Association Publishing, 2006.

6 Hay P, Chinn D, Forbes D, et al. Royal Australian and New Zealand College of Psychiatrists clinical practice guidelines for the treatment of eating disorders. Aust N Z J Psychiatry 2014;48:977-1008.

7 Garber AK, Michihata N, Hetnal K, et al. A prospective examination of weight gain in hospitalized adolescents with anorexia nervosa on a recommended refeeding protocol. J Adolesc Health 2012;50:24-9.

8 Garber AK, Mauldin K, Michihata N, et al. Higher calorie diets increase rate of weight gain and shorten hospital stay in hospitalized adolescents with anorexia nervosa. J Adolesc Health 2013;53:579-84.

9 Higa T, Okura H, Imai K, et al. Refeeding syndrome in a patient with anorexia nervosa. J Am Coll Cardiol 2013;62:19.

10 Boateng AA, Sriram K, Meguid MM, et al. Refeeding syndrome: treatment considerations based on collective analysis of literature case reports. Nutrition 2010;26:156-67.

11 Rio A, Whelan K, Goff L, et al. Occurrence of refeeding syndrome in adults started on artificial nutrition support: prospective cohort study. BMJ Open 2013;3:e002173.

12 Garber AK, Sawyer SM, Golden NH, et al. A systematic review of approaches to refeeding in patients with anorexia nervosa. Int $\mathrm{J}$ Eat Disord 2016;49:293-310.
13 Royal College of Psychiatrists, Royal College of Physicians. MARSIPAN: management of really sick patients with anorexia nervosa (College report CR189). 2nd edn. Royal College of Psychiatrists, 2014.

14 Golden NH, Katzman DK, et al, Society for Adolescent Health and Medicine. Position paper of the Society for adolescent health and medicine: medical management of restrictive eating disorders in adolescents and young adults. J Adolesc Health 2015;56:121-5.

15 Cape Town Metropole Paediatric Interest Group. Refeeding syndrome: guidelines, 2009. Available: http://www.adsa.org.za/ Portals/14/Documents/Clinical20Guidelines20Refeeding20Syndrom e20Paeds20Section200nly20_.pdf [Accessed 16 Nov 2017].

16 Afzal NA, Addai S, Fagbemi A, et al. Refeeding syndrome with enteral nutrition in children: a case report, literature review and clinical guidelines. Clin Nutr 2002;21:515-20.

17 Academy for Eating Disorders. A guide to medical care; Critical points for early recognition \& medical risk management in the care of individuals with eating disorders. 3rd edn. Academy for Eating Disorders, 2016.

18 Redgrave GW, Coughlin JW, Schreyer CC, et al. Refeeding and weight restoration outcomes in anorexia nervosa: challenging current guidelines. Int J Eat Disord 2015;48:866-73.

19 Gentile MG, Pastorelli P, Ciceri R, et al. Specialized refeeding treatment for anorexia nervosa patients suffering from extreme undernutrition. Clin Nutr 2010;29:627-32.

20 O'Connor G, Goldin J. The refeeding syndrome and glucose load. Int $J$ Eat Disord 2011;44:182-5.

21 Abbott nutrition. Available: https://nutrition.abbott/au/product/ ensure-plus-hn/nutrition [Accessed 16 Nov 2017].

22 Whitelaw M, Gilbertson H, Lam P-Y, et al. Does aggressive refeeding in hospitalized adolescents with anorexia nervosa result in increased hypophosphatemia? J Adolesc Health 2010;46:577-82.

23 Parker EK, Faruquie SS, Anderson G, et al. Higher caloric refeeding is safe in hospitalised adolescent patients with restrictive eating disorders. J Nutr Metab 2016;2016:1-9.

24 Ornstein RM, Golden NH, Jacobson MS, et al. Hypophosphatemia during nutritional rehabilitation in anorexia nervosa: implications for refeeding and monitoring. J Adolesc Health 2003;32:83-8.

25 O'Connor G, Nicholls D, Hudson L, et al. Refeeding low weight hospitalized adolescents with anorexia nervosa: a multicenter randomized controlled trial. Nutr Clin Pract 2016;31:681-9.

26 Maginot TR, Kumar MM, Shiels J, et al. Outcomes of an inpatient refeeding protocol in youth with anorexia nervosa: Rady children's Hospital San Diego/University of California, San Diego. J Eat Disord 2017;5:1.

27 Smith K, Lesser J, Brandenburg B, et al. Outcomes of an inpatient refeeding protocol in youth with anorexia nervosa and atypical anorexia nervosa at children's hospitals and clinics of Minnesota. $J$ Eat Disord 2016;4:35.

28 Golden $\mathrm{NH}$, Keane-Miller C, Sainani KL, et al. Higher caloric intake in hospitalized adolescents with anorexia nervosa is associated with reduced length of stay and no increased rate of refeeding syndrome. $J$ Adolesc Health 2013;53:573-8.

29 Agostino H, Erdstein J, Di Meglio G. Shifting paradigms: continuous nasogastric feeding with high caloric intakes in anorexia nervosa. $J$ Adolesc Health 2013;53:590-4.

30 Madden S, Miskovic-Wheatley J, Clarke S, et al. Outcomes of a rapid refeeding protocol in adolescent anorexia nervosa. $J$ Eat Disord 2015;3:8.

31 Chan A-W, Tetzlaff JM, Altman DG, et al. Statement: defining standard protocol items for clinical trials. Ann Intern Med 2013;2013:200-7.

32 American Psychiatric Association. Diagnostic and statistical manual of mental disorders. 5th ed. Arlington, VA: American Psychiatric Publishing, 2013.

33 Detsky AS, McLaughlin JR, Baker JP, et al. What is subjective global assessment of nutritional status? JPEN J Parenter Enteral Nutr 1987;11:8-13.

34 Bishop CW, Bowen PE, Ritchey SJ. Norms for nutritional assessment of American adults by upper arm anthropometry. Am J Clin Nutr 1981;34:2530-9.

35 Addo OY, Himes JH. Reference curves for triceps and subscapular skinfold thicknesses in US children and adolescents. Am J Clin Nutr 2010;91:635-42.

36 Staun M, Pironi L, Bozzetti F, et al. Muscular weakness assessment: use of normal isometric strength data. The National isometric muscle strength (NIMS) database Consortium Archives PF physical medicine. Rehabilitation 1996;77:1251-5. 